\title{
Doppler ultrasound assessment in women with threatened abortion
}

\author{
Gustavo Romero-Gutiérrez ${ }^{1}$, Antonio Abraham Huebe-Martínez ${ }^{2}$, Immer Amaral-Navarro ${ }^{1}$, \\ Armando Saúl Ruiz-Treviño 1
}

${ }^{1}$ Dept. Obstetrics and Gynecology. UMAE. Hospital de Gineco-Pediatría No 48, IMSS, León, Guanajuato. México

${ }^{2}$ Dept. Radiology. Hospital of Family Medicine N 51, IMSS, León, Guanajuato, México

\section{Email address:}

gustavoromerog@hotmail.com (G. Romero-Gutiérrez), antonio.huebe@imss.gob.mx (A. A. Huebe-Martínez), anaponceponce@hotmail.com (I. Amaral-Navarro), armando.ruiz.t@gmail.com (A. Ruiz-Treviño)

\section{To cite this article:}

Gustavo Romero-Gutiérrez, Antonio Abraham Huebe-Martínez, Immer Amaral-Navarro, Armando Saú Ruiz-Treviño. Doppler Ultrasound Assessment in Women with Threatened Abortion, Clinical Medicine Research. Vol. 2, No. 3, 2013, pp.24-28.

doi: $10.11648 /$ j.cmr.20130203.11

\begin{abstract}
Objective: To determine which variables obtained by Doppler ultrasound are associated with the outcome of patients with threatened abortion. Study design: We carried out a cross-sectional study. The patient population included patients with pregnancy less than 20 weeks, who were hospitalized with the diagnosis of threatened abortion. Results: We followed the outcome of 223 patients and found 32 patients (14.3\%) who aborted and 191 patients (85.7\%) who did not abort. The fetal heart rate for the group who aborted was significantly lower than the group who did not abort (143 $\pm 2.9 \mathrm{vs.} 154 \pm$ 3.6 beats per minute, respectively), $\mathrm{p}=0.002$; the resistance index of uterine artery was higher $(0.79 \pm 0.07 \mathrm{vs} .0 .76 \pm 0.09$, respectively), $\mathrm{p}=0.018$; and the resistance index of trophoblastic flow was higher ( $0.55 \pm 0.04$ vs. $0.50 \pm 0.06$, respectively), $\mathrm{p}=0.041$. The resistance index of umbilical artery and corpus luteum were not statistically different. Conclusion: Alterations of fetal heart rate, resistance index of uterine artery and trophoblastic flow are associated with adverse outcome in women with threatened abortion.
\end{abstract}

Keywords: Doppler Ultrasound, Threatened Abortion, Resistance Index, Uterine Artery, Trophoblastic Flow

\section{Introduction}

The clinical features of threatened abortion are vaginal bleeding before 20 weeks of gestation, accompanied by cramping pain, and sometimes without changes of the cervix [1].

Other than clinical symptoms, ultrasound examination is an important auxiliary procedure for diagnosis of abortion. The sonographic findings using conventional ultrasound have been assessed and are considered to have a prognostic value that interacts with other clinical and maternal factors analyzed [2,3]. Some parameters obtained using conventional ultrasound considered as prognostic factors are: the morphological characteristics of the yolk sac, adequate visualization of the embryo by transvaginal technique, changes in fetal heart rate and macroscopic lesions of the placenta $[3,4]$.

The introduction of Doppler ultrasound in obstetrics has allowed evaluating hemodynamic characteristics from the first trimester of pregnancy. For example, Kurjak et al., [5], in one of the first studies using transvaginal pulsed-wave Doppler ultrasound in early pregnancies, identified the uterine arteries in $100 \%$ of the patients.

The flow in the peritrophoblastic area was visualized in 94\% women; blood flow in the umbilical artery and fetal aorta was detected since seventh week; and the flow blood of the corpus luteum was identified in $75 \%$ patients.

The measures obtained by Doppler ultrasonography that may have prognostic value to the evolution of pregnancy include uteroplacental blood flow, also known as trophoblastic flow. Jaffe et al. reported that abnormal Doppler findings were associated with a significantly higher prevalence of complicated pregnancies, among women with abnormal Doppler findings, $43 \%$ ended in miscarriage, whereas among women with normal findings only $1.4 \%$ of women miscarried [6].

In a study by Alcázar et al. [7] assessment of the flow of the corpus luteum showed differences in patients with normal pregnancies compared to patients with abortion. A higher mean resistance index was observed in women with 
abortion than in control group. Recently, El-Mashad et al. [8] reported that the abnormal resistance of uterine artery may be useful to predict recurrent fetal loss. However, Pellizzari et al. [9] did not find differences in Doppler velocimetry of the uterine arteries in women with normal and pathological pregnancy.

There remains controversy as to whether Doppler ultrasound assessment is useful in predicting outcomes of women with threatened abortion. The aim of this study was to determine which blood flow measurements are associated with poor outcome in these women.

\section{Methods}

A cross-sectional study was conducted at the Hospital of Obstetrics and Gynecology at the Mexican Institute of Social Security in Leon, Mexico. Recruitment was carried out from patients who were hospitalized with the diagnosis of threatened abortion. The study protocol was approved by the ethics and research committee of the local institutional review board, and the women who agreed to participate in the study completed a written informed consent form.

By a sampling of consecutive cases, we included patients who presented to our hospital between 5 to 20 weeks of pregnancy and were diagnosed with threatened abortion. The patients were recruited according to the following criteria:

Inclusion criteria: patients with pregnancy between 5 to 20 weeks of gestation, who were diagnosed with threatened abortion according to the institutional standards of our hospital.

Exclusion criteria: patients with multiple pregnancies, molar pregnancy, ectopic pregnancy, amenorrhea with different etiologies of pregnancy, maternal history of systemic diseases and uterine anatomic abnormalities.

Elimination criteria: patients who did not have their outcome data through week 20 of gestation due to relocation.

We evaluated both socio-demographic variables and clinical features of our population. The socio-demographic variables registered included: maternal age, defined as completed years at the time of delivery; education level, registered as the number of completed years of school; income, registered in U.S.A. dollars; previous pregnancies, including abortions, and gestational weeks at the moment of ultrasound assessment. Also clinical factors such as medical conditions and obstetric complications were included such as the presence of pelvic pain, vaginal bleeding and cervical features (closed or dilated). These parameters were coded as 1 when present and 0 when absent.

Afterward, a transvaginal ultrasound examination was carried out with a high color Doppler resolution transvaginal ultrasound, using standard techniques. Hewlett Packard M2 410B, serial number US99601963; option number ABM $\mathrm{H} 24$ (Andover, Massachusetts); equipped with a 5-MHz transvaginal probe. The high pass filter was set at $100 \mathrm{~Hz}$ and the spatial peak temporal average was $80 \mathrm{~mW} / \mathrm{cm} 2$ (according to the limits of security recommended by the
American Food and Drug Administration for use in fetal medicine). The color Doppler application was used only to localize the vessels, but the measurement of all parameters were calculated based on velocities obtained by pulsed-wave Doppler.

The ultrasonographic assessment was performed with the patient in the decubitus position to avoid supine hypotension; the patient was not in labor and it was performed a prior measurement of blood pressure in order just to confirm normal values of this vital sign.

A two-dimensional, B-mode scan was first performed to evaluate the state of the pregnancy and to determine the true gestational age by measuring the fetal crown-rump length, in women with first trimester pregnancy; and fetal biparietal diameter and femoral length were measured in women at 13 to 20 weeks of gestation. Flow velocity waveforms were measured and results were analyzed by calculating the resistance index (RI). This evaluation was obtained from the umbilical cord; the systolic velocity of the wave-forms and the diastolic velocity of the wave-forms were determined; the resistance index resulted from the following formula: $\mathrm{RI}=\mathrm{S}-\mathrm{D} / \mathrm{S}$; where $\mathrm{RI}$ is the resistance index, $\mathrm{S}$ corresponded to the systolic velocity and D corresponded to the diastolic velocity [10].

The Doppler ultrasound variables that were measured included: fetal heart rate (FHR), with normal values ranging from 90 to 160 beats per minute [4]; RI of uterine arteries, with normal range considered at a resistance index value lower than 0.55 [11]; RI of the trophoblastic or implantation flow, with normal considered at a RI value lower than 0.55 [6]; RI of the corpus luteum, considering as normal values a RI lower than 0.64 [12]; and RI of the umbilical artery, with normal value considered at a RI value under 0.58 [13]. In order to eliminate interobserver variability, Doppler examinations were performed by one physician (GRG), and repeated measurements were performed in $10 \%$ of patients to determine the intraobserver concordance coefficient, which must be over $89 \%$ [14].

After the ultrasound assessment, the patient continued with the recommendations by the assigned physician and the outcome was documented until 20 weeks of gestation.

\section{Statistical analysis}

Data were analyzed using descriptive statistical analysis, which included arithmetic mean, standard error (SE) and percentages values of the categorical data. The groups were compared using the Chi-square analysis or Fisher Exact' test for categorical variables; and Student $t$ test or Mann-Whitney U test for continuous variables as appropriate depending on their normal distribution. The alpha value was set at 0.05 .

\section{Results}

234 patients were recruited with threatened abortion, 11 of them were eliminated because they had not completed the evolution to 20 weeks of gestation, resulting in 223 (95.2\%) patients in the final sample. 
The mean gestational age in which the ultrasound examination was performed was $9.36 \pm 0.3$ (range 5-18 weeks). The follow-up to the twentieth week was carried out by noting the date on which they were contacted. It was found that of the 223 patients affected by threatened abortion 32 (14.3\%) aborted, and 191 women (85.7\%) continued their pregnancy to full term.

Table 1, shows the demographic characteristics of the patient population utilized in this study. The mean age of the women who aborted was $26.5 \pm 1.0$, and $25.5 \pm 1.4$ years for the group who did not abort, $\mathrm{p}=0.843$; the average years of schooling were $8.7 \pm 0.5$ and $9.2 \pm 0.2$, respectively, $\mathrm{p}=$ 0.486 ; and average family monthly income were 388.1 and 367.1 dollars, respectively, $\mathrm{p}=0.633$. There were no statistical differences between the groups in clinical variables as noted in Table 1.

Table 1. Socio-demographic and clinical variables in both groups.

\begin{tabular}{llll}
\hline Variable & $\begin{array}{l}\text { Women with } \\
\text { abortion } \\
(\mathbf{n}=\mathbf{3 2})\end{array}$ & $\begin{array}{l}\text { Women } \\
\text { without abortion } \\
(\mathbf{n}=\mathbf{1 9 1})\end{array}$ & $\mathbf{P}$ \\
\hline Maternal age & $26.5 \pm 1.0$ & $25.5 \pm 1.4$ & 0.843 \\
Scholarship (years) & $8.7 \pm 0.5$ & $9.2 \pm 0.2$ & 0.486 \\
Income (dollars) & 388.1 & 367.1 & 0.633 \\
Number of pregnancies & 2.8 & 2.6 & 0.552 \\
Abortions previous & $5(15)$ & $53(27.7)$ & 0.358 \\
Vaginal bleeding & $30(93)$ & $141(73.8)$ & 0.464 \\
Pelvic pain & $24(75)$ & $128(67)$ & 0.812 \\
Closed cérvix & $30(93)$ & $183(95)$ & 0.958 \\
\hline
\end{tabular}

Mean $\pm S E ; n(\%)$.

The ultrasound Doppler measurements are described in table 2, the average fetal heart rate in the group who aborted was $143 \pm 2.9$ beats per minute and in the group who did not abort was $154 \pm 3.6, \mathrm{p}=0.002$; the mean uterine artery resistance index was $0.79 \pm 0.07$ and $0.76 \pm 0.09$, respectively, $\mathrm{p}=0.018$; the mean trophoblastic flow resistance index was $0.55 \pm 0.04$ and $0.50 \pm 0.06$, respectively, $\mathrm{p}$ $=0.041$; the other Doppler measurements did not have statistical difference.

Table 2. Ultrasound Doppler wave-form measurements in both groups.

\begin{tabular}{lllc}
\hline Variable & $\begin{array}{l}\text { Women } \\
\text { with } \\
\text { abortion } \\
\text { (n= 32) }\end{array}$ & $\begin{array}{l}\text { Women } \\
\text { abortion } \\
(\mathbf{n}=\mathbf{1 9 1})\end{array}$ & without \\
\hline FHR & $143 \pm 2.9$ & $154 \pm 3.6$ & 0.002 \\
Corpus luteum RI & $0.58 \pm 0.06$ & $0.61 \pm 0.04$ & 0.325 \\
Umbilical artery RI & $0.89 \pm 0.05$ & $0.90 \pm 0.08$ & 0.543 \\
Uterine artery RI & $0.79 \pm 0.07$ & $0.76 \pm 0.09$ & 0.018 \\
Trophoblastic flow RI & $0.55 \pm 0.04$ & $0.50 \pm 0.06$ & 0.041 \\
\hline
\end{tabular}

FHR: Fetal heart rate; RI: Resistance index.

\section{Discussion}

Threatened abortion is a common complication during pregnancy. It has been reported that it affects up to $15-20 \%$ of patients, and the risk of ending in abortion is multiplied 2.6 times [1]. In the present study, from all patients that were attended to in our hospital with threatened abortion, $14.3 \%$ ended in abortion.

The maternal mean age was similar in both groups, this contrast with some studies that have shown an increase risk of abortion occurrence with increased age [3]. By studying the socio-demographic variables we found no significant differences. For example, scholarship ranged from eight to nine years and the family monthly income varied from 367 to 388 dollars.

Similarly none of the obstetric and gynecological antecedents were statistically significant, for example the number of pregnancies ranged from 2.6 to 2.8 and the number of previous abortions varied from $15 \%$ to $27.7 \%$.

Regarding the clinical variables as presence of pain and bleeding or cervical features (closed or dilated), we found no significant difference. The absence of this difference supports the fact that in some cases of abortion, the bleeding is not a prognostic factor, as it has been classically considered [1]. Moreover, recently it has been reported that the detection rate of miscarriage in screening by ultrasound findings was $85.7 \%$ and by vaginal bleeding was only $45 \%$ [3].

Of the ultrasound variables obtained by measure of the fetal heart rate (FHR) and Doppler velocimetry, we found one patient with a FHR below 100 beats per minute, who later had an abortion. Most authors who have analyzed the FHR observed that bradycardia is associated with an increased risk of spontaneous abortion. For example, Benson et al. [4] demonstrated a poor outcome in patients with FHR less than 90 beats per minute. More recent, Hamela-Olkowska et al. [15] evaluated the embryonic and fetal heart rates with similar results. In our study we also found a significantly lower fetal heart rate in patients who aborted. We consider that the FHR should be evaluated as soon as possible in all pregnancies, because regardless of the presence of symptoms in the patient, is a consistent variable in the outcome of the pregnancy.

We did not find differences in the resistance index of the corpus luteum or the resistance index of the umbilical artery in women who aborted and those who did not. This result is similar as reported by Alcazar et al. [7], who found no difference in the vessels of the corpus luteum in women with threatened abortion as compared with the control group; so both findings support the fact that measurements of these vessels have no utility in women with threatened abortion.

On the other hand, the usefulness of the evaluation of the uterine artery with Doppler ultrasound in pregnancy has been widely addressed. We now know that their alterations are useful in the diagnosis of preeclampsia, intrauterine growth restriction and other pregnancy morbidities [16]. In the women studied, the measurement of RI of uterine artery was altered, and had statistical significance for adverse pregnancy outcomes; it also was found mostly higher in patients who suffered abortion, similar to that reported by 
Cooley et al [16]. Moreover, in another study Iacovella et al. [17], reported that altered measurement of the uterine artery evaluated by Doppler ultrasound at first trimester was associated with late stillbirth after 34 weeks of gestation.

In contrast to our results, Pellizzari et al. [9] found no statistical significance of this variable, by comparing the values in patients with normal pregnancies and those complicated by uterine bleeding. These results may be due to the small sample size and that they only included patients in the first trimester. It is likely that the vascular disorders due to abnormal placentation are present from the beginning of pregnancy, but these are only detected by Doppler up to the second trimester. While the authors found no clinical utility, they concluded that color Doppler sonography plays an important role in the understanding of the different pathophysiological aspects of the uteroplacental circulation [9].

Although Alcázar et al. [18] found no significant difference in the uteroplacental circulation in patients with threatened abortion; in our study we found that the measurement of resistance index of trophoblastic or uteroplacental flow was statistically significant being a consistent Doppler variable that might be predictor of adverse outcome. Altered trophoblastic flow is due to inadequate trophoblastic invasion of spiral arteries in the decidua; sonographic and morphological studies of early abortions have shown an abnormal blood flow in the uteroplacental circulation in women who suffer an abortion [19]. This agrees with our Doppler ultrasound assessment, with the finding of a rise in the level of the resistance index of trophoblastic flow, in the group who had an abortion compared with those with normal development at 20 weeks of gestation; these findings are similar to those reported previously by Jaffe and his group of researchers [6].

In a recent study Yazawa et al. [20], reported a higher incidence of uterine vascular malformations, in particular in the patients after abortion, and they recommend performing follow-up analysis using Doppler ultrasonography. Similarly, we suggest carrying out this procedure for patients with threatened abortion. This will guide us on the likely prognosis and outcome of pregnancy; and when necessary we will be able to inform patients of the higher risk of abortion occurrence.

\section{Conclusion}

Altered fetal heart rate, resistance index of uterine artery and trophoblastic flow, assessed with Doppler ultrasonography, are associated with adverse outcome in women with threatened abortion.

\section{References}

[1] Sotiriadis A, Papatheodorou S, Makrydimas G. Threatened miscarriage: evaluation and management. BMJ 2004; 329:152-155.
[2] Bae S, Karnitis J. Triple ultrasound markers including fetal cardiac activity are related to miscarriage risk. Fertil Steril 2011; 96:1145-1148.

[3] Papaioannou GI, Syngelaki A, Maiz N, Ross JA, Nicolaides KH. Ultrasonographic prediction of early miscarriage. Hum Reprod 2011; 26:1685-1692.

[4] Benson CB, Doubilet PM. Slow embryonic heart rate in early first trimester: indicator of poor pregnancy outcome. Radiology 1994; 192:343-344.

[5] Kurjak A, Crvenkovic G, Salihagic A, Zalud I, Miljan M. The assessment of normal early pregnancy by transvaginal color Doppler ultrasonography. J Clin Ultrasound 1993; 21:3-8.

[6] Jaffe R, Dorgan A, Abramowicz JS. Color Doppler imaging of the uteroplacental circulation in the first trimester: value in predicting pregnancy failure or complication. ARJ Am J Roentgenol 1995; 164:1255-1258.

[7] Alcázar JL, Laparte C, López-Garcia G. Corpus luteum blood flow in abnormal early pregnancy. J Ultrasound Med 1996; 15:645-649.

[8] El-mashad AI, Mohamed MA, Farag MA, Ahmad MK, Ismail Y. Role of uterine artery Doppler velocimetry indices and plasma adrenomedullin level in women with unexplained recurrent pregnancy loss. J Obstet Gynaecol Res 2011; 37:51-57.

[9] Pellizzari P, Pozzan C, Marchiori S, Zen T, Gangemi M. Assessment of uterine artery blood flow in normal first-trimester pregnancies and in those complicated by uterine bleeding. Ultrasound Obstet Gynecol 2002; 19:366-370.

[10] Goslin RG, King DH. Ultrasound angiology. In: Marcus AW, Adamson L. Editors. Arteries and veins. Edimburg: Churchill-Livingstone, 1975: 61-98.

[11] Leible S, Cumsille F, Walton R, Muñoz H, Jankelevich J, Sepulveda W. Discordant uterine artery velocity waveforms as a predictor of subsequent miscarriage in early viable pregnancies. Am J Obstet Gynecol 1998; 179:1587-1593.

[12] Salim A, Zalud I, Farmakides G, Shulman H, Kurjak A, Latin V. Corpus luteum blood flow in normal and abnormal early pregnancy: evaluation with transvaginal color and pulsed Doppler sonography. J Ultrasound Med 1994; 13:971-975.

[13] Arduini D, Rizzo G. Umbilical artery velocity waveforms in early pregnancy: A transvaginal color Doppler study. J Clin Ultrasound 1991; 19:335-339.

[14] Alcázar JL. Intraobserver variability of pulsatility index measurements in three fetal vessels in the first trimester. $\mathrm{J}$ Clin Ultrasound 1997; 25:366-371.

[15] Hamela-Olkowska A, Wiech K, Jalinik K, Zarviewski D, Kornatowski L, Dangel J. Evaluation of the embryonic and foetal heart rate at $6(+0)$ to $11(+6)$ weeks of gestation. Ginekol Pol 2009; 80:188-192.

[16] Cooley SM, Donnelly JC, Walsh T, MacMahon C, Gillan J, Geary MP. The impact of umbilical and uterine artery Doppler indices on antenatal course, labor and delivery in a low risk primigravid population. J Perinat Med 2011; 39:143-149.

[17] Iacovella C, Franchi M, Egbor M, Bhide A, Thilaganathan B. Relationship of first-trimester uterine artery Doppler to late 
stillbirth. Prenat Diagn 2012; 17:1-5.

[18] Alcázar JL, Ruiz-Pérez ML. Uteroplacental circulation in patients with first-trimester threatened abortion. Fertil Steril $2000 ; 73: 130-5$.

[19] Jauniaux E, Johns J, Burton GJ. The role of ultrasound imaging in diagnosing and investigating early pregnancy failure.
Ultrasound Obstet Gynecol 2005; 25:613-624.

[20] Yazawa H, Soeda S, Hiraiwa T, Takaiwa M, Hasegawa-Endo S, Kojima M, Fujimori K. Prospective evaluation of the incidence of uterine vascular malformations developing after abortion or delivery. J Minim Invasive Gynecol 2013. pii: S1553-4650(12)01381-7. doi: 10.1016/j.jmig.2012.12.008. [Epub ahead of print]. 